\title{
Experiences of LGBTIQ+ Population within Healthcare Contexts*
}

\section{Editorial}

\author{
CRISTIANO SCANDURRA \\ cristiano.scandurra@unina.it \\ University of Naples Federico II
}

\author{
PAOLO VALERIO \\ paolo.valerio@unina.it \\ University of Naples Federico II
}

Although research on the health of lesbian, gay, bisexual, transgender, intersex, queer (LGBTIQ+) populations ${ }^{1}$ is expanding rapidly, including a focus on social and health policies, research on the experiences of LGBTIQ+ people within healthcare contexts needs to be further developed and expanded. Indeed, social acceptance of LGBTIQ+ people has been improving in the last decades, but they continue to face stigma and discrimination (Gallup, 2016; Grant et al., 2011; Scandurra et al., 2017). Stigmatising experiences, combined with a lack of access to culturally-affirming healthcare, result in a disadvantaged social status and health disparities for LGBTIQ+ population (Fredriksen-Goldsen et al., 2014; Reisner et al., 2014).

To this end, many LGBTIQ+ people still avoid healthcare contexts for fear of discrimination from healthcare providers or because they feel the lack of healthcare professional knowledge and negative views of LGBTIQ+ individuals (Krehely, 2009). For instance, approximately 30\% of LGBTIQ+ adults do not have access to healthcare services or lack a regular healthcare provider (Winter, 2012). Furthermore, LGBTIQ+ people are more likely to avoid healthcare contexts compared to heterosexual counterparts, delaying proper treatments and, thus, resulting in adverse health outcomes (Shields et al., 2012).

Despite subjective experiences of LGBTIQ+ people within healthcare contexts, structural barriers (e.g., binary male/female medical record identification system, financial barriers, lack of equality signs and gender-neutral language, etc.) significantly contribute to the delay or avoidance of seeking healthcare (Roberts and Fantz, 2014). Indeed, the structural/institutional stigma is embedded within some health organisations due to the existence of laws and regulations that do not guarantee full equality for LGBTIQ+ populations. On the other hand, many healthcare providers still have little knowledge of the specific health needs of this

\footnotetext{
* We thank David M. Frost, University College London, for his fundamental support in the first stages of this special issue.

${ }^{1}$ The acronym LGBTIQ+ is used here to refer to the lesbian, gay, bisexual, transgender, intersexual and queer populations. The plus at the end of the acronym is meant to encompass other individuals, such as asexual, non-binary, pansexual, genderqueer, questioning individuals. No form of disrespect or erasure is intended here by not specifically mentioning other types of initialisms or terms from different languages and cultures. The editors have adopted the acronym LGBTIQ+ because they feel it represents the most inclusive, concise and comprehensive way to envisage this ever-growing community.
} 
population (Roberts and Fantz, 2014), leading to non-affirmative and competent provisions of care. Therefore, a more accurate understanding of the quality of care received and perceived by LGBTIQ+ population represents a fundamental social issue and a central focus for reducing health inequalities. It means that it is urgent to provide affirmative and inclusive healthcare services for LGBTIQ+ people.

The current thematic issue of the puntOorg International Journal intends to provide possible answers to this need by focusing on the experiences of LGBTIQ+ people within healthcare contexts. To this end, we were able to collect scientific contributions from multidisciplinary viewpoints.

More specifically, the contribution by Annalisa Anzani (University of Milano Bicocca, Italy) reviews recent literature on microaggressions toward transgender and gender nonconforming (TGNC) individuals. The critical review offered by Annalisa Anzani is mainly focused on the emotional, cognitive and behavioural reactions to microaggressions, as well as on the detrimental effects that microaggressions towards TGNC people have on their health and well-being. The work ends with some reflections on the affirmative psychological practice with TGNC individuals.

The research by Emanuele Maria Merlo, Fabio Frosinone, and Salvatore Settineri (University of Messina, Italy) addresses TGNC experiences again within healthcare contexts, but from a different perspective. Indeed, the authors discuss their psychological-clinical experiences with transgender people who have access to the gender identity clinic of the University Hospital G. Martino in Messina (Italy), paying specific attention to the 'clinical contact' from a theoretical perspective which adopts both the phenomenology and the life-course framework.

The paper by Andrea Pennasilico and Anna Lisa Amodeo (University of Naples Federico II, Italy) critically reviews recent literature on bisexual health. In particular, the authors present a critical review on 3 main areas: (1) stigma towards bisexual population; (2) health of bisexual people and its interface with stigma; and (3) experiences of bisexual people within healthcare contexts.

The article by Joshua Minh Quach (New York University, US) explores qualitatively the lived-experiences of 20 men who have sex with men (MSM), divided in two age-cohorts (1849 and 50+ ages). Specifically, the author provides an interesting comparison between these cohorts concerning their patient-provider relationships and assessing differences and similarities in healthcare experiences with regard to social trauma, medical trauma, and their proximity to the 1980s AIDS crisis.

Finally, the contribution by Davide Bizjak (University of Naples Federico II, Italy) addresses inclusive organisational practices for TGNC people from an organisational perspective, proposing a model centred on the relationship between transition stages and work status. This model may be applied to healthcare contexts if interpreted as complex organisations.

The editors hope this collection of scientific papers will expand upon previously published work on the topic of healthcare experiences of LGBTIQ+ individuals, drawing the attention of both scholars and policymakers. Indeed, this thematic issue highlights the need for promoting culturally-affirming healthcare for LGBTIQ+ people, removing both individual and structural barriers which produce health disparities and drastically increase the psycho-social risk of developing negative health outcomes. 


\section{Reference list}

Fredriksen-Goldsen, K.I., Simoni, J.M., Kim, H.J., Lehavot, K., Walters, K.L., Yang, J., HoyEllis, C.P., and Muraco, A. (2014), "The Health Equity Promotion Model: Reconceptualization of Lesbian, Gay, Bisexual, and Transgender (LGBT) Health Disparities", American Journal of Orthopsychiatry, 84 (6): 653-663.

Gallup (2016), “Gay and Lesbian Rights”. Available online at www.gallup.com/poll/1651/gaylesbian-rights.aspx (last accessed: November 9, 2019).

Grant, J.M., Mottet, L.A., Tanis, J., Harrison, J., Herman, J.L., and Keisling, M. (2011), Injustice at Every Turn: A Report of the National Transgender Discrimination Survey. Washington, DC: National Center for Transgender Equality \& National Gay and Lesbian Task Force. Available online at www.transequality.org/issues/national-transgenderdiscriminationsurvey-full-report (last accessed: November 9, 2019).

Krehely, J. (2009), "How to Close the LGBT Health Disparities Gap", Center for American Progress: $1-9$.

Reisner, S.L., White, J.M., Bradford, J.B., and Mimiaga M.J. (2014), “Transgender Health Disparities: Comparing Full Cohort and Nested Matched-Pair Study Designs in a Community Health Center", LGBT Health, 1 (3): 177-184.

Roberts, T.K., and Fantz, C.R. (2014), "Barriers to Quality Health Care for the Transgender Population", Clinical Biochemistry, 47 (10/11): 983-987.

Scandurra, C., Amodeo, A.L., Valerio, P., Bochicchio, V., and Frost, D.M. (2017), "Minority Stress, Resilience, and Mental Health: A Study of Italian Transgender People", Journal of Social Issues, 73 (3): 563-585.

Shields, L., Zappia, T., Blackwood, D., Watkins, R., Wardrop, J., and Chapman, R. (2012), "Lesbian, Gay, Bisexual, and Transgender Parents Seeking Health Care for Their Children: A Systematic Review of the Literature", Worldviews on Evidence-Based Nursing, 9 (4): 200-209.

Winter, C. (2012), "Health Equity Series: Responding to LGBT Health Disparities", Missouri Foundation for Health Publication. Available online at https://mffh.org/wordpress/wpcontent/uploads/2016/04/LGBTHealthEquityReport.pdf (last accessed: November 9, 2019). 